\title{
An efficient green synthesis of dispirohydroquinolines via a diastereoselective one-pot eight-component reaction
}

\author{
Sajjad Salahi a, Malek Taher Maghsoodlou a,* , Nourallah Hazeri a, Mojtaba Lashkari a , \\ Santiago Garcia-Granda b ${ }^{\text {, Laura Torre-Fernandez }}{ }^{\text {b }}$ \\ a Department of Chemistry, Faculty of Science, University of Sistan and Baluchestan, P. O. Box 98135-674 Zahedan, Iran \\ ${ }^{\mathrm{b}}$ Department of Physical and Analytical Chemistry, University of Oviedo-CINN, Oviedo, Spain
}

\section{A R T I C L E I N F O}

Article history:

Received 25 January 2015

Accepted 19 March 2015

Published 20 July 2015

Keywords:

Eight-component reaction

Dispirohydroquinoline

Meldrum's acid

Citric acid

\section{A B S T R A C T}

The one-pot eight-component reaction between Meldrum's acid, an aromatic aldehyde, and an aryl amine was achieved in the presence of citric acid catalyst. The corresponding dispirohydroquinolines were obtained in good yields with excellent diastereoselectivity. This method is a combination of the Knoevenagel and Michael reactions.

(C) 2015, Dalian Institute of Chemical Physics, Chinese Academy of Sciences. Published by Elsevier B.V. All rights reserved.

\section{Introduction}

Green chemistry involves intrinsic atom economy, helps to save energy and reduce waste, allows easy work up and avoids hazardous chemicals [1-9]. The development of a simple, eco-friendly reaction protocol for the synthesis of highly functionalized medicinal compound libraries is an attractive area of research [10-12]. In this context, multi-component reactions (MCRs) have become interesting [13-16], where three or more starting materials react in one-pot to form a complex product. The product must contain at least part of the substrate and the starting materials should be commercially available in large quantities or easy to prepare. The MCR is convergent, offers operational simplicity and facile automation, has atom and step economy [17] and generates minimal waste. MCR chemistry can also be used to synthesize heterocycles [18].

As part of our continuing research [19-25], herein we report a green, mild, high yielding one-pot eight-component reac- tion for the synthesis of a new heterocyclic system from Meldrum's acid 1, an aromatic aldehyde $\mathbf{2}$ and an aryl amine $\mathbf{3}$ in the presence of citric acid (Scheme 1).

\section{Experimental}

\subsection{General methods}

Melting points (m.p.) and infrared (IR) spectra were measured on an Electrothermal 9100 instrument and a JASCO FT/IR-460 plus spectrometer, respectively. The ${ }^{1} \mathrm{H}$ and ${ }^{13} \mathrm{C}$ NMR spectra were recorded on a Bruker DRX-400 Avance spectrometer with $\mathrm{CDCl}_{3}$ as the solvent at 400 and $100 \mathrm{MHz}$, respectively. All reagents were purchased from Merck (Darmastadt, Germany) or Fluka (Buchs, Switzerland), and used without further purification.

\subsection{General procedure for the synthesis of}

\footnotetext{
* Corresponding author. Tel: +98-5412446565; Fax: +98-5412446565; E-mail: mt_maghsoodlou@chem.usb.ac.ir, mt_maghsoodlou@yahoo.com DOI: 10.1016/S1872-2067(15)60846-4 | http://www.sciencedirect.com/science/journal/18722067 | Chin. J. Catal., Vol. 36, No. 7, July 2015
} 


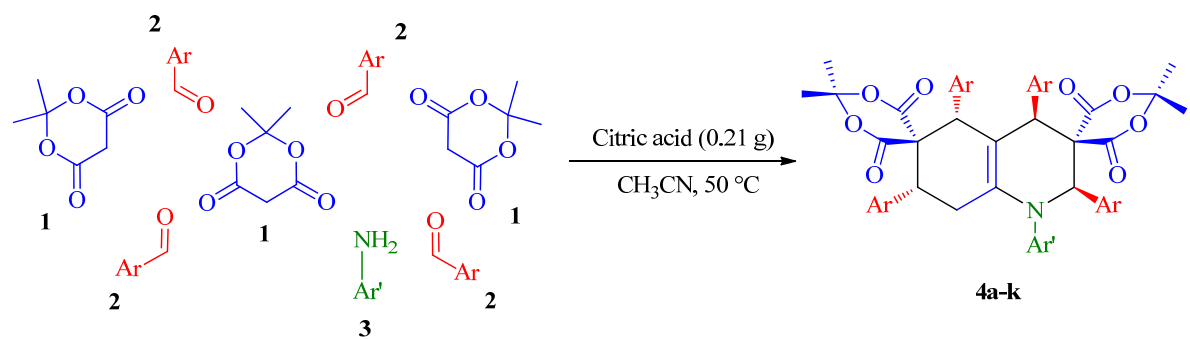

Scheme 1. Synthesis of dispirohydroquinolines via a one-pot eight-component reaction.

\section{dispirohydroquinolines}

To a solution of Meldrum's acid (3.0 mmol), aldehyde (4.0 $\mathrm{mmol})$ and aniline $(1.0 \mathrm{mmol})$ in acetonitrile $(5 \mathrm{~mL})$, citric acid $(0.21 \mathrm{~g})$ was added and magnetically stirred at $50{ }^{\circ} \mathrm{C}$. The reaction progress was monitored by TLC. After $24 \mathrm{~h}$, the resultant precipitates were collected by filtration and washed with acetonitrile $(3 \times 2 \mathrm{~mL})$.

Spectral data of selected new products are as follows.

$1^{\prime}, 2^{\prime}, 4^{\prime}, 5^{\prime}, 7^{\prime}$-Pentaphenyl-1'H-dispiro[2', $2^{\prime}, 5^{\prime}, 7^{\prime}, 8^{\prime}$-tetrahydroquinoline-5,3':6',5'-bis(2,2-dimethyl [1,3]dioxane-4,6-dione)] (4a). White solid; ${ }^{1} \mathrm{H} \mathrm{NMR}\left(\mathrm{CDCl}_{3}, 400 \mathrm{MHz}\right): \delta=0.36,0.38,0.60$ and $0.62(4 \mathrm{~s}, 12 \mathrm{H}, 4 \mathrm{Me}), 2.55-2.66\left(\mathrm{~m}, 2 \mathrm{H}, \mathrm{H}^{\prime}, \mathrm{H}^{\prime \prime}-8^{\prime}\right), 4.02(\mathrm{dd}$ $\left.1 \mathrm{H}, J=11.6 \mathrm{~Hz}, J=6.0 \mathrm{~Hz}, \mathrm{H}-7^{\prime}\right), 4.65$ and 4.67 (2s, 2H, H-4', H-5') $5.26\left(\mathrm{~s}, 1 \mathrm{H}, \mathrm{H}-2^{\prime}\right), 6.04\left(\mathrm{~d}, 1 \mathrm{H}, J=7.6, \mathrm{H}_{\mathrm{Ar}}\right), 6.08(\mathrm{~d}, 1 \mathrm{H}, J=$ 7.6, $\left.\mathrm{H}_{\mathrm{Ar}}\right), 6.71\left(\mathrm{~d}, 1 \mathrm{H}, J=7.6 \mathrm{~Hz}, \mathrm{H}_{\mathrm{Ar}}\right), 6.75\left(\mathrm{~d}, 1 \mathrm{H}, J=8.0 \mathrm{~Hz}, \mathrm{H}_{\mathrm{Ar}}\right.$ ), 7.00-7.58 (m, 21H, $\left.\mathrm{H}_{\text {Ar }}\right)$.

$1^{\prime}$-(4-Methoxyphenyl)-2', 4', $5^{\prime}, 7^{\prime}$-tetra(4-methylphenyl)-1'Hdispiro[2', $4^{\prime}, 5^{\prime}, 7^{\prime}, 8^{\prime}$-tetrahydroquinoline-5,3': $6^{\prime}, 5^{\prime \prime}$-bis(2,2-dime thyl[1,3] dioxane-4,6-dione)] (4b). White solid; ${ }^{1} \mathrm{H}$ NMR $\left(\mathrm{CDCl}_{3}\right.$, $400 \mathrm{MHz}$ ): $\delta=0.40,0.43,0.65$ and $0.66(4 \mathrm{~s}, 12 \mathrm{H}, 4 \mathrm{Me}), 2.19$, 2.23 and 2.25 (3s, 12H, 4ArMe), 2.44-2.67 (m, 2H, $\left.\mathrm{H}^{\prime}, \mathrm{H}^{\prime \prime}-8^{\prime}\right)$, 3.71 (s, 3H, OMe), 3.95 (dd, $1 \mathrm{H}, J=12.0 \mathrm{~Hz}, J=5.6 \mathrm{~Hz}, \mathrm{H}-7^{\prime}$ ), 4.55 and 4.59 (2s, 2H, H-4', H-5'), 5.15 (s, 1H, H-2'), 5.94 (d, 1H, $\left.J=8.0 \mathrm{~Hz}, \mathrm{H}_{\mathrm{Ar}}\right), 5.97\left(\mathrm{~d}, 1 \mathrm{H}, J=8.0 \mathrm{~Hz}, \mathrm{H}_{\mathrm{Ar}}\right), 6.55(\mathrm{t}, 2 \mathrm{H}, J=7.2$, $\left.\mathrm{H}_{\mathrm{Ar}}\right), 6.67-7.48\left(\mathrm{~m}, 16 \mathrm{H}, \mathrm{H}_{\mathrm{Ar}}\right)$.

$1^{\prime}$-(Phenyl)-2', 4', $5^{\prime}, 7^{\prime}$-tetra(2-methylphenyl-1'H-dispiro[2', $4^{\prime}, 5^{\prime}, 7^{\prime}, 8^{\prime}$-tetrahydro-quinoline-5,3':6', $5^{\prime \prime}$-bis(2,2-dimethyl[1,3] dioxane-4,6-dione)] (4c). White solid; ${ }^{1} \mathrm{H} \mathrm{NMR}\left(\mathrm{CDCl}_{3}, 400\right.$ $\mathrm{MHz}$ ): $\delta=0.48,0.50,1.15$ and $1.17(4 \mathrm{~s}, 12 \mathrm{H}, 4 \mathrm{Me}), 0.92,2.32$ and 2.51 (3s, 12H, 4ArMe), 2.52 (dd, $1 \mathrm{H}, J=14.4,5.6 \mathrm{~Hz}, \mathrm{H}^{\prime}-8^{\prime}$ ), 2.65-2.73 (m, 1H, H"-8'), 4.32 (dd, $1 \mathrm{H}, J=12.0 \mathrm{~Hz}, J=5.2 \mathrm{~Hz}$, H-7'), 4.76 and 4.82 (2s, 2H, H-4', H-5'), 5.59 (s, 1H, H-2'), 6.71-7.84 (m, 21H, $\left.\mathrm{H}_{\mathrm{Ar}}\right)$.

$1^{\prime}$-(4-Chlorophenyl)-2', 4', 5', $7^{\prime}$-tetra(4-methylphenyl)-1' $\mathrm{H}$ -

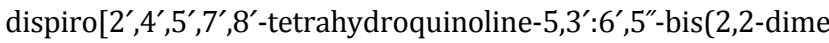
thyl[1,3] dioxane-4,6-dione)] (4e). White solid; ${ }^{1} \mathrm{H}$ NMR $\left(\mathrm{CDCl}_{3}\right.$, $400 \mathrm{MHz}$ ): $\delta=0.40,0.43,0.66$ and 0.67 (4s, 12H, 4Me), 2.21, 2.23 and 2.26 (3s, 12H, 4ArMe), 2.47-2.64 (m, 2H, $\left.\mathrm{H}^{\prime}, \mathrm{H}^{\prime \prime}-8^{\prime}\right)$, 3.95 (dd, $\left.1 \mathrm{H}, J=12.0 \mathrm{~Hz}, J=5.6 \mathrm{~Hz}, \mathrm{H}^{-} 7^{\prime}\right), 4.55$ and $4.58(2 \mathrm{~s}, 2 \mathrm{H}$, $\left.\mathrm{H}-4^{\prime}, \mathrm{H}-5^{\prime}\right), 5.14\left(\mathrm{~s}, 1 \mathrm{H}, \mathrm{H}-2^{\prime}\right), 5.96(\mathrm{t}, 2 \mathrm{H}, J=8.0 \mathrm{~Hz}, \mathrm{HAr}), 6.56(\mathrm{t}$, $\left.2 \mathrm{H}, J=7.2 \mathrm{~Hz}, \mathrm{H}_{\mathrm{Ar}}\right), 6.88-7.42\left(\mathrm{~m}, 16 \mathrm{H}, \mathrm{H}_{\mathrm{Ar}}\right)$.

$1^{\prime}$-(4-Fluorophenyl)-2', $4^{\prime}, 5^{\prime}, 7^{\prime}$-tetra(4-methoxyphenyl)-1' ${ }^{\prime} \mathrm{H}$ -

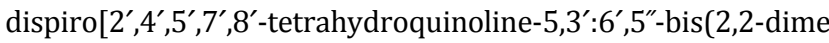
thyl[1,3]dioxane-4,6-dione)] (4j). White solid; m.p. $247-248^{\circ} \mathrm{C}$; IR (KBr): $v$ 1767, 1730, 1652, 1610, 1509, 1462, 1381, 1302,
1245, $1035 \mathrm{~cm}^{-1} ;{ }^{1} \mathrm{H}$ NMR $\left(\mathrm{CDCl}_{3}, 400 \mathrm{MHz}\right): \delta=0.46,0.49,0.70$ and $0.75(4 \mathrm{~s}, 12 \mathrm{H}, 4 \mathrm{Me}), 2.45(\mathrm{dd}, 1 \mathrm{H}, J=17.2 \mathrm{~Hz}, J=5.6 \mathrm{~Hz}$, $\mathrm{H}^{\prime}-8^{\prime}$ ), 2.53-2.60 (m, 1H, H"-8'), 3.70, 3.72 and 3.73 (3s, 12H, 4 $\mathrm{MeO}$ ), 3.92 (dd, 1H, J = 12.0, J = 5.6 Hz, H-7'), 4.51, 4.53 (2s, 2H, H-4' ${ }^{\prime}$ H-5'), 5.10 (s, 1H, H-2'), 5.99-6.04 (m, 2H, $\mathrm{H}_{\mathrm{Ar}}$ ), 6.31-6.35 $\left(\mathrm{m}, 1 \mathrm{H}, \mathrm{H}_{\mathrm{Ar}}\right), 6.63\left(\mathrm{dd}, 1 \mathrm{H}, J=8.8 \mathrm{~Hz}, J=2.8 \mathrm{~Hz}, \mathrm{H}_{\mathrm{Ar}}\right.$ ), 6.71 (dd, $\left.1 \mathrm{H}, J=8.8 \mathrm{~Hz}, J=2.8 \mathrm{~Hz}, \mathrm{H}_{\mathrm{Ar}}\right), 6.75-7.01\left(\mathrm{~m}, 9 \mathrm{H}, \mathrm{H}_{\mathrm{Ar}}\right), 6.71(\mathrm{dd}$, $\left.1 \mathrm{H}, J=7.2 \mathrm{~Hz}, J=2.4 \mathrm{~Hz}, \mathrm{H}_{\mathrm{Ar}}\right), 7.37(\mathrm{dd}, 1 \mathrm{H}, J=8.4 \mathrm{~Hz}, J=2.0 \mathrm{~Hz}$, $\mathrm{H}_{\mathrm{Ar}}$ ), 7.44 (dd, $\left.1 \mathrm{H}, J=8.4 \mathrm{~Hz}, J=2.0 \mathrm{~Hz}, \mathrm{H}_{\mathrm{Ar}}\right) ;{ }^{13} \mathrm{C} \mathrm{NMR}\left(\mathrm{CDCl}_{3}\right.$, $100 \mathrm{MHz}$ ): $\delta=28.1,28.3,28.6,28.7$ (4Me), 32.0 (C-8'), 46.8, 49.8 and 52.2 (C-4', C-5', C-7'), 55.1, 55.2 and 55.3 (40Me), 61.7 and 61.9 (C-3', C-6'), 69.7 (C-2'), 103.3 (C-4'a), 105.2 and 105.5 $\left(2 \mathrm{CMe}_{2}\right), 112.77,113.4,113.6,113.7,113.8,114.0,114.3,127.7$, 128.2, 129.3, 129.7, 130.2, 130.3, 130.4, 130.5, 132.0, 132.2, 132.6, $140.6\left(\mathrm{~d}, J_{\mathrm{CF}}=2.9 \mathrm{~Hz}\right), 141.9\left(\mathrm{C}_{\mathrm{Ar}}, \mathrm{C}-8^{\prime} \mathrm{a}\right), 158.7,158.8$, 159.2 and $159.4\left(4 \mathrm{C}_{\mathrm{Ar}}-0\right), 160.7\left(\mathrm{~d}, J_{\mathrm{CF}}=245.7 \mathrm{~Hz}, \mathrm{C}_{\mathrm{Ar}}-\mathrm{F}\right), 162.1$, $164.3,168.4$ and $169.8(4 \mathrm{C}=0)$.

$1^{\prime}$-(4-Bromophenyl)-2', 4', $5^{\prime}, 7^{\prime}$-tetra(2-methylphenyl)-1'Hdispiro[ $\left[2^{\prime}, 4^{\prime}, 5^{\prime}, 7^{\prime}, 8^{\prime}\right.$-tetrahydroquinoline-5,3': $6^{\prime}, 5^{\prime \prime}$-bis(2,2-dime thyl[1,3] dioxane-4,6-dione)] (4k). White solid; m.p. 230-232 ${ }^{\circ} \mathrm{C}$; IR (KBr): $v$ 1768, 1736, 1664, 1513, 1486, 1392, 1290, 1069 $\mathrm{cm}^{-1}$; ${ }^{1} \mathrm{H}$ NMR $\left(\mathrm{CDCl}_{3}, 400 \mathrm{MHz}\right): \delta=0.40,0.43,0.66$ and 0.68 (4s, 12H, 4Me), 2.22, 2.23 and 2.26 (3s, 12H, 4 ArMe), 2.47-2.60 (m, 2H, H', H'-8'), 3.95 (dd, 1H, J = 11.6 Hz, J = 5.6 Hz, H-7'), 4.55 and $4.58\left(2 \mathrm{~s}, 2 \mathrm{H}, \mathrm{H}-4^{\prime}, \mathrm{H}-5^{\prime}\right), 5.13\left(\mathrm{~s}, 1 \mathrm{H}, \mathrm{H}-2^{\prime}\right), 5.95(\mathrm{t}, 2 \mathrm{H}, J=$ $\left.8.0 \mathrm{~Hz}, \mathrm{H}_{\mathrm{Ar}}\right), 6.55\left(\mathrm{t}, 2 \mathrm{H}, J=6.8 \mathrm{~Hz}, \mathrm{H}_{\mathrm{Ar}}\right), 6.88-7.41\left(\mathrm{~m}, 16 \mathrm{H}, \mathrm{H}_{\mathrm{Ar}}\right)$; ${ }^{13} \mathrm{C} \mathrm{NMR}\left(\mathrm{CDCl}_{3}, 100 \mathrm{MHz}\right): \delta=20.9,20.9,21.0$ and 21.1 (4ArMe), 27.9, 28.2, 28.4 and 28.5 (4Me), 32.9 (C-8'), 47.2, 50.2 and 52.6 (C-4', C-5', C-7'), 61.6 and 61.7 (C-3', C-6'), 69.7 (C-2'), 103.2 (C-4'a), 105.2 and 105.4 (2CMe $), 120.0,128.3,128.4$, 128.6, 128.8, 129.0, 129.1, 129.1, 129.2, 129.3, 130.8, 131.2, 131.5, 131.6, 131.8, 132.9, 133.9, 135.4, 136.6, 136.8, 137.7, 138.3, 141.5, 143.9 ( $\left.\mathrm{C}_{\text {Ar, }} \mathrm{C}-8^{\prime} \mathrm{a}\right), 162.0,164.2,168.2$ and 169.6 $(4 \mathrm{C}=0)$.

Benzylidene Meldrum's acid (18). White solid; m.p. 120$122{ }^{\circ} \mathrm{C} ;{ }^{1} \mathrm{H} \mathrm{NMR}\left(\mathrm{CDCl}_{3}, 400 \mathrm{MHz}\right): \delta=1.84(\mathrm{~s}, 6 \mathrm{H}, 2 \mathrm{Me}), 7.51(\mathrm{t}$, $2 \mathrm{H}, J=8.0 \mathrm{~Hz}, \mathrm{HPh}$ ), $7.59(\mathrm{t}, 1 \mathrm{H}, J=8.0 \mathrm{~Hz}, \mathrm{HPh}), 8.08(\mathrm{~d}, 2 \mathrm{H}, J=$ $8.0 \mathrm{~Hz}, \mathrm{HPh}$ ), 8.46 (s, 1H, Hvinylic).

Bis-adduct (19). White solid; m.p. $180-182{ }^{\circ} \mathrm{C} ;{ }^{1} \mathrm{H}$ NMR $\left(\mathrm{CDCl}_{3}, 400 \mathrm{MHz}\right): \delta=1.71$ and $1.84(2 \mathrm{~s}, 6 \mathrm{H}, 2 \mathrm{Me}), 4.64-4.70$ (m, 3H, CHbenzylic, 2CHMeldrum's acid), 7.30-7.59 (m, 5H, HPh).

\section{Results and discussion}

The one-pot eight-component reaction of Meldrum's acid, benzaldehyde and aniline was chosen as a model to optimize 
Table 1

Optimization of amount of catalyst in the synthesis of dispirohydroquinoline 4a.

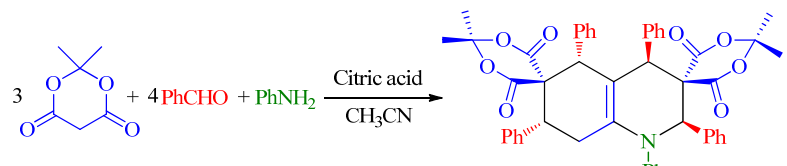

$\mathrm{Ph}$

\begin{tabular}{lcccc}
\hline Entry & Catalyst $(\mathrm{g})$ & $T\left({ }^{\circ} \mathrm{C}\right)$ & Time $(\mathrm{h})$ & Isolated yield $(\%)$ \\
\hline 1 & 0.10 & 30 & 100 & 64 \\
2 & 0.15 & 30 & 72 & 69 \\
3 & 0.15 & 50 & 50 & 73 \\
4 & 0.21 & 30 & 48 & 70 \\
5 & 0.21 & 40 & 41 & 76 \\
6 & 0.21 & 50 & 32 & 84 \\
7 & 0.21 & 75 & 24 & 79 \\
8 & 0.21 & 100 & 24 & trace \\
9 & 0.31 & 30 & 23 & 75 \\
10 & 0.42 & 50 & 20 & 81 \\
11 & 200 & 30 & 22 & 82 \\
\hline
\end{tabular}

the conditions in the presence of different quantities of citric acid catalyst at various temperatures. The best yield of corresponding dispirohydroquinoline (84\%) was observed when $0.21 \mathrm{~g}$ citric acid was used at $50^{\circ} \mathrm{C}$ (Table 1 , entry 6 ).

We propose that this transformation involves eight components. Product 4 occurs from the condensation of three moles of Meldrum's acid, four moles of aldehyde and one mole of aniline. The reaction is diastereoselective and generates four new stereogenic centers. Quantum mechanical calculations confirmed this observation [12].
Table 2

Synthesis of structure of dispiro[tetrahydroquinoline-bis(2,2-dimethyl [1,3]dioxane-4,6-dione)] derivatives $4 \mathbf{a}-\mathbf{k}$.

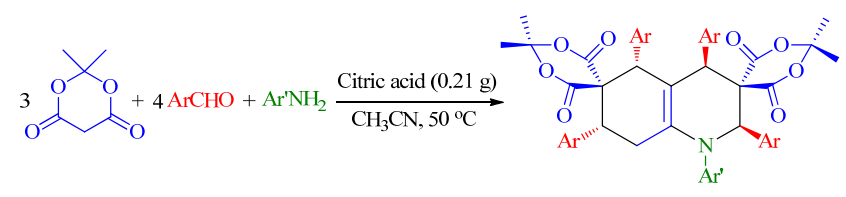

\begin{tabular}{lccccc}
\hline Entry & $\mathrm{Ar}$ & $\mathrm{Ar}^{\prime}$ & Product & Isolated yield (\%) & Ref. \\
\hline 1 & $\mathrm{Ph}$ & $\mathrm{Ph}$ & $\mathbf{4 a}$ & 84 & {$[24]$} \\
2 & $4-\mathrm{MeC}_{6} \mathrm{H}_{4}$ & $4-\mathrm{MeOC}_{6} \mathrm{H}_{4}$ & $\mathbf{4 b}$ & 80 & {$[24]$} \\
3 & $2-\mathrm{MeC}_{6} \mathrm{H}_{4}$ & $\mathrm{Ph}$ & $\mathbf{4 c}$ & 65 & {$[24]$} \\
4 & $4-\mathrm{MeC}_{6} \mathrm{H}_{4}$ & $4-\mathrm{FC}_{6} \mathrm{H}_{4}$ & $\mathbf{4 d}$ & 74 & {$[24]$} \\
5 & $4-\mathrm{MeC}_{6} \mathrm{H}_{4}$ & $4-\mathrm{ClC}_{6} \mathrm{H}_{4}$ & $\mathbf{4 e}$ & 77 & {$[24]$} \\
6 & $4-\mathrm{MeC}_{6} \mathrm{H}_{4}$ & $4-\mathrm{BrC}_{6} \mathrm{H}_{4}$ & $\mathbf{4 f}$ & 81 & {$[24]$} \\
7 & $4-\mathrm{MeOC}_{6} \mathrm{H}_{4}$ & $4-\mathrm{BrC}_{6} \mathrm{H}_{4}$ & $\mathbf{4 g}$ & 82 & {$[25]$} \\
8 & $2-\mathrm{ClC}_{6} \mathrm{H}_{4}$ & $\mathrm{Ph}_{4}$ & $\mathbf{4 h}$ & 72 & {$[23]$} \\
9 & $\mathrm{Ph}_{4}$ & $4-\mathrm{MeOC}_{6} \mathrm{H}_{4}$ & $\mathbf{4 i}$ & 79 & {$[23]$} \\
\hline
\end{tabular}

* All known products reported previously in the literature were characterized by comparison of m.p., IR and NMR spectra with those of authentic samples.

The chemical structures of all new compounds (Table 2) were confirmed by IR, ${ }^{1} \mathrm{H}$ and ${ }^{13} \mathrm{C}$ NMR spectroscopy and elemental analysis. The relative stereochemistry of these compounds was confirmed by single-crystal X-ray crystallography (Scheme 2) [23-25] and the stereochemistry was determined by comparison of spectroscopic data with those of authentic samples (Table 3).

The suggested mechanism for this eight-component reaction is presented in Scheme 3 and is based on the Knoevenagel and Michael reactions. First, Knoevenagel condensation occurs

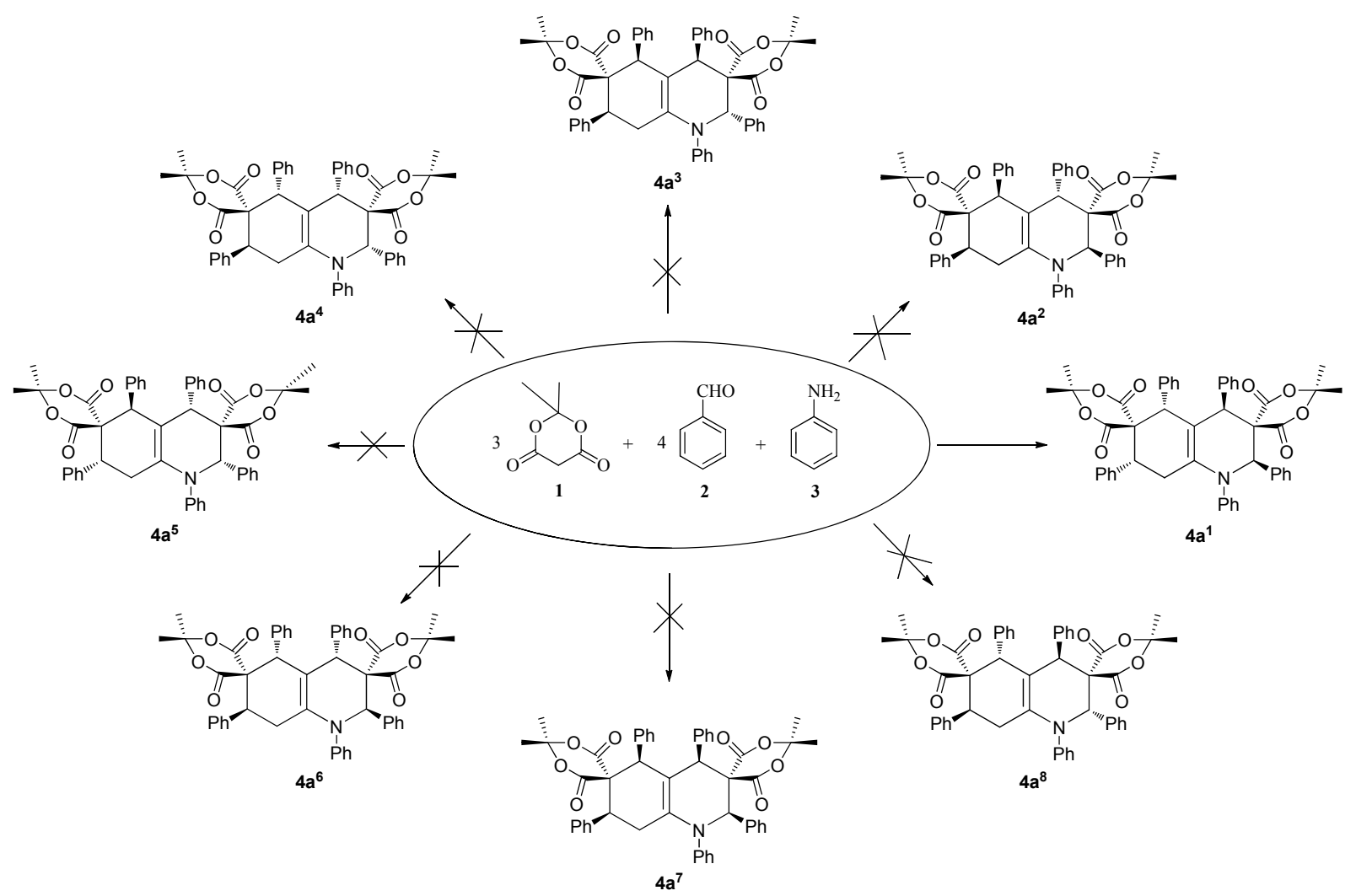

Scheme 2. Structures of eight possible diastereomers 4 a. 
Table 3

Comparison of ${ }^{1} \mathrm{H}$ NMR data.

\begin{tabular}{|c|c|c|c|}
\hline Entry & Product & H shift (found) & H shift [24] \\
\hline 1 & $4 a$ & $\begin{array}{l}4 \times \text { Me: } 0.36,0.38,0.60 \text { and } 0.62(4 \mathrm{~s}) ; \mathrm{H}^{\prime}, \mathrm{H}^{\prime \prime}-8^{\prime}: 2.55-2.66(\mathrm{~m}) \\
\mathrm{H}-7^{\prime}: 4.02(\mathrm{dd}, J=11.6 \mathrm{~Hz}, J=6.0 \mathrm{~Hz}) ; \mathrm{H}-4^{\prime}, \mathrm{H}^{\prime} 5^{\prime}: 4.65 \text { and } 4.67 \\
(2 \mathrm{~s}) ; \mathrm{H}^{\prime}: 5.26(\mathrm{~s})\end{array}$ & $\begin{array}{l}4 \times \mathrm{Me}: 0.37,0.39,0.61 \text { and } 0.64(4 \mathrm{~s}) ; \mathrm{H}^{\prime}-8^{\prime}: 2.59(\mathrm{dd}, J=17.2 \mathrm{~Hz} \text {, } \\
J=5.6 \mathrm{~Hz}) ; \mathrm{H}^{\prime \prime}-8^{\prime}: 2.64-2.71(\mathrm{~m}) ; \mathrm{H}-7^{\prime}: 4.03(\mathrm{dd}, J=11.6 \mathrm{~Hz}, J= \\
6.0 \mathrm{~Hz}) ; \mathrm{H}-4^{\prime}, \mathrm{H}^{\prime} 5^{\prime}: 4.66 \text { and } 4.69(2 \mathrm{~s}) ; \mathrm{H}-2^{\prime}: 5.27(\mathrm{~s})\end{array}$ \\
\hline 2 & $4 b$ & $\begin{array}{l}4 \times \text { Me: } 0.40,0.43,0.65 \text { and } 0.66(4 \mathrm{~s}) ; 4 \times \text { ArMe: } 2.19,2.23 \text { and } \\
2.25(3 \mathrm{~s}) ; \mathrm{H}^{\prime}, \mathrm{H}^{\prime \prime}-8^{\prime}: 2.44-2.67(\mathrm{~m}) ; \text { OMe: } 3.71(\mathrm{~s}) ; \mathrm{H}-7^{\prime}: 3.95 \\
(\mathrm{dd}, J=12.0 \mathrm{~Hz}, J=5.6 \mathrm{~Hz}) ; \mathrm{H}-4^{\prime}, \mathrm{H}-5^{\prime}: 4.55 \text { and } 4.59(2 \mathrm{~s}) ; \\
\mathrm{H}-2^{\prime}: 5.15(\mathrm{~s})\end{array}$ & 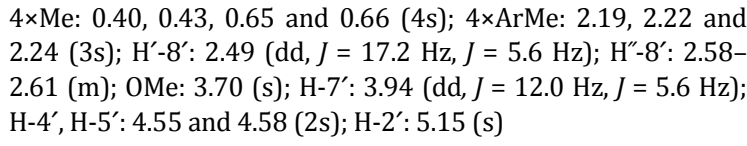 \\
\hline 3 & $4 c$ & $\begin{array}{l}4 \times \text { Me: } 0.48,0.50,1.15 \text { and } 1.17(4 \mathrm{~s}) ; 4 \times \text { ArMe: } 0.92,2.32 \text { and } \\
2.51(3 \mathrm{~s}) ; \mathrm{H}^{\prime}-8^{\prime}: 2.52(\mathrm{dd}, J=14.4,5.6 \mathrm{~Hz}) ; \mathrm{H}^{\prime \prime}-8^{\prime}: 2.65-2.73 \\
(\mathrm{~m}) ; \mathrm{H}-7^{\prime}: 4.32(\mathrm{dd}, J=12.0 \mathrm{~Hz}, J=5.2 \mathrm{~Hz}) ; \mathrm{H}^{\prime} 4^{\prime}, \mathrm{H}-5^{\prime}: 4.76 \text { and } \\
4.82(2 \mathrm{~s}) ; \mathrm{H}-2^{\prime}: 5.59(\mathrm{~s})\end{array}$ & $\begin{array}{l}4 \times \mathrm{Me}: 0.48,0.50,1.15 \text { and } 1.17(4 \mathrm{~s}) ; 4 \times A r M e: 0.92,2.26 \text { and } \\
2.51 \quad(3 \mathrm{~s}) ; \mathrm{H}^{\prime} \text { and } \mathrm{H}^{\prime \prime}-8^{\prime}: 2.46-2.68(\mathrm{~m}) ; \mathrm{H}-7^{\prime}: 4.31(\mathrm{dd}, J=12.0 \\
\mathrm{Hz}, J=5.2 \mathrm{~Hz}) ; \mathrm{H}-4^{\prime}, \mathrm{H}^{\prime}-5^{\prime}: 4.76 \text { and } 4.82(2 \mathrm{~s}) ; \mathrm{H}-2^{\prime}: 5.59(\mathrm{~s})\end{array}$ \\
\hline 4 & $4 e$ & $\begin{array}{l}4 \times \text { Me: } 0.40,0.43,0.66 \text { and } 0.67(4 \mathrm{~s}) ; 4 \times \text { ArMe: } 2.21,2.23 \text { and } \\
2.26(3 \mathrm{~s}) ; \mathrm{H}^{\prime} \text { and } \mathrm{H}^{\prime \prime}-8^{\prime}: 2.47-2.64(\mathrm{~m}) ; \mathrm{H}^{\prime}: 3.95(\mathrm{dd}, J=12.0 \\
\mathrm{Hz}, J=5.6 \mathrm{~Hz}) ; \mathrm{H}-4^{\prime}, \mathrm{H}^{\prime}-5^{\prime}: 4.55 \text { and } 4.58(2 \mathrm{~s}) ; \mathrm{H}-2^{\prime}: 5.14(\mathrm{~s})\end{array}$ & $\begin{array}{l}4 \times \mathrm{Me}: 0.40,0.43,0.66 \text { and } 0.67(4 \mathrm{~s}) ; 4 \times \text { ArMe: } 2.21,2.23 \text { and } \\
2.25(3 \mathrm{~s}) ; \mathrm{H}^{\prime} \text { and } \mathrm{H}^{\prime \prime}-8^{\prime}: 2.47-2.64(\mathrm{~m}) ; \mathrm{H}-7^{\prime}: 3.94(\mathrm{dd}, J=12.0 \\
\mathrm{Hz}, J=5.6 \mathrm{~Hz}) ; \mathrm{H}-4^{\prime}, \mathrm{H}^{\prime}-5^{\prime}: 4.55 \text { and } 4.58(2 \mathrm{~s}) ; \mathrm{H}-2^{\prime}: 5.13(\mathrm{~s})\end{array}$ \\
\hline 5 & $4 j$ & $\begin{array}{l}4 \times \text { Me: } 0.46,0.49,0.70 \text { and } 0.75(4 \mathrm{~s}) ; \mathrm{H}^{\prime}-8^{\prime}: 2.45(\mathrm{dd}, J=17.2 \\
\mathrm{Hz}, J=5.6 \mathrm{~Hz}) ; \mathrm{H}^{\prime \prime}-8^{\prime}: 2.53-2.60(\mathrm{~m}) ; 4 \times \mathrm{OMe}: 3.70,3.72 \text { and } \\
3.73(3 \mathrm{~s}) ; \mathrm{H}-7^{\prime}: 3.92(\mathrm{dd}, J=12.0, J=5.6 \mathrm{~Hz}) ; \mathrm{H}^{\prime} 4^{\prime}, \mathrm{H}-5^{\prime}: 4.51 \\
\text { and } 4.53 ;(2 \mathrm{~s}) \mathrm{H}-2^{\prime}: 5.10(\mathrm{~s})\end{array}$ & \\
\hline 6 & $4 k$ & $\begin{array}{l}4 \times \text { Me: } 0.40,0.43,0.66 \text { and } 0.68(4 \mathrm{~s}) ; 4 \times A r M e: 2.22,2.23 \text { and } \\
2.26(3 \mathrm{~s}) ; \mathrm{H}^{\prime}, \mathrm{H}^{\prime \prime}-8^{\prime}: 2.47-2.60(\mathrm{~m}, 2 \mathrm{H}) ; \mathrm{H}^{\prime}: 7^{\prime}: 3.95(\mathrm{dd}, J=11.6 \\
\mathrm{Hz}, J=5.6 \mathrm{~Hz}) ; \mathrm{H}-4^{\prime}, \mathrm{H}^{\prime} 5^{\prime}: 4.55 \text { and } 4.58(2 \mathrm{~s}) ; \mathrm{H}-2^{\prime}: 5.13(\mathrm{~s})\end{array}$ & \\
\hline
\end{tabular}

between benzaldehyde and Meldrum's acid to generate 6 [26]. This intermediate then decomposes to acetone and 8 [27]. Acetone condenses with aniline to give imine $\mathbf{9}$ and tautomerizes to enamine 10, which reacts with the aldehyde to produce reactive Barbas dienamine 12 (2-amino-1,3-butadiene) [27-37]. Barbas dienamine undergoes a double Michael addition with the Knoevenagel product to furnish enamine 14 [32]. Then, 14 is added to 6 [36-38] and generates the dispiro[tetrahydroquinoline-bis(2,2-dimethyl[1,3] dioxane-4,6-dione)] derivatives. This hypothesis is supported by the mechanistic investigation of proline-catalyzed spirotrione's formation by reaction of an aldehyde and Meldrum's acid with enones reported by Barbas et al. [27,39].

During the preparation of product 4a, 18 (Knoevenagel condensation product) and bis-adduct 19 (competing Michael addition of Meldrum's acid with the resulting benzylidene) were isolated from the mixture of reaction as byproducts (Scheme 4) [40].

\section{Conclusions}

We have reported a one-pot eight-component reaction for the synthesis of dispirohydroquinolines by reacting Meldrum's acid, an aldehyde and aniline. Mild reaction conditions, good isolated yields, short reaction time and use of a green catalyst are the remarkable advantages of this method. The products have four stereocenters and are synthesized in a diastereoselective manner.
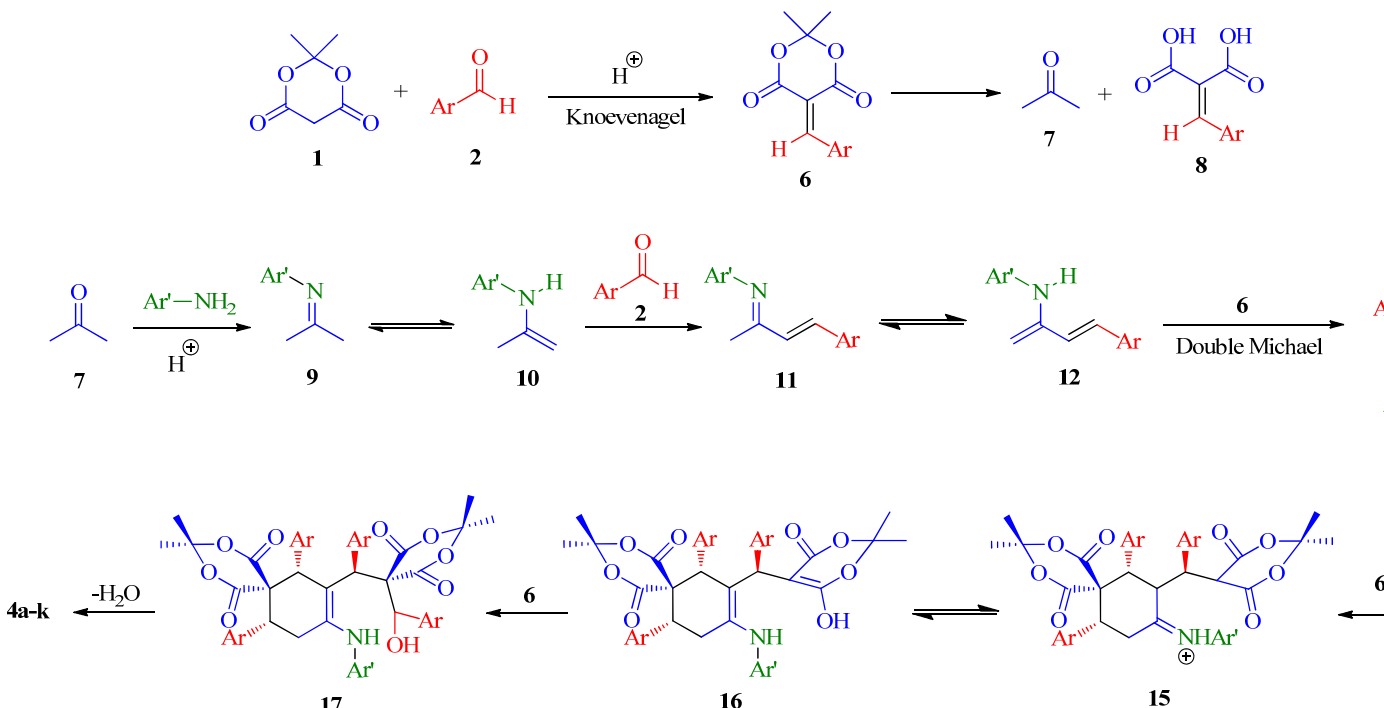

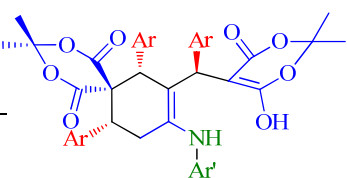

16

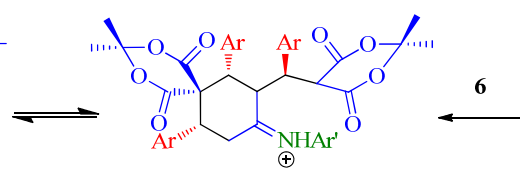

15
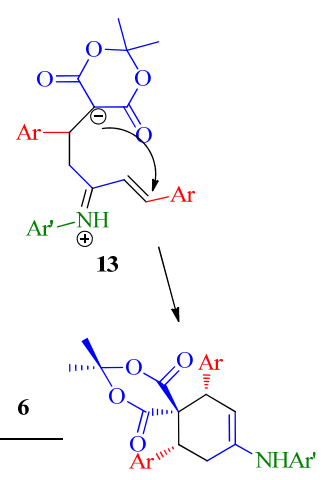

14

Scheme 3. Suggested mechanism for the synthesis of dispirohydroquinolines. 


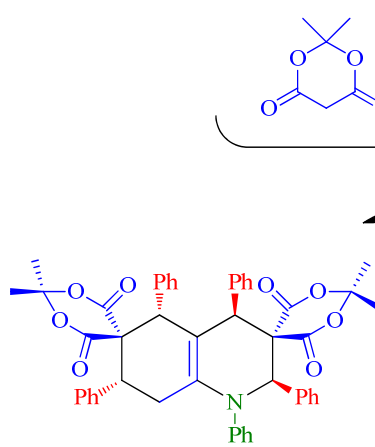

$4 a$
$+\mathrm{PhCHO}+\mathrm{PhNH}_{2}$

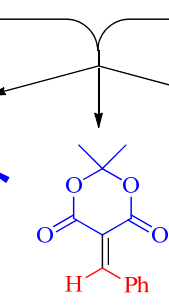

18

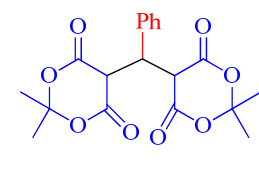

19
Scheme 4. Generation of byproducts in this reaction.

\section{Acknowledgments}

We gratefully acknowledge the funding support received for this project from the Research Council of the University of Sistan and Baluchestan. This work was partially supported by FEDER funding, the Spanish Ministerio de Economía y Competitividad MAT2006-01997, MAT2010-15094 and the Factoría de Cristalización (Consolider Ingenio 2010).

\section{References}

[1] Kumar A, Gupta G, Srivastava S. Green Chem, 2011, 13: 2459

[2] Kumar A, Gupta M K, Kumar M. Green Chem, 2012, 14: 290

[3] Tanaka K, Sugino T, Toda F. Green Chem, 2000, 2: 303

[4] Raston C L, Scott J L. Green Chem, 2000, 2: 49

[5] Anastas P T, Warner J C. Green Chemistry: Theory and Practice. Oxford: Oxford University Press, 1998

[6] McNulty J, Das P. EurJ Org Chem, 2009: 4031

[7] McNulty J, Das P. Tetrahedron Lett, 2009, 50: 5737

[8] McNulty J, Das P, McLeod D. Chem Eur J, 2010, 16: 6756

[9] Das P, McLeod D, McNulty J. Tetrahedron Lett, 2011, 52: 199

[10] Dömling A. Chem Rev, 2006, 106: 17

[11] Zhu J P, Bienayme H. Multicomponent Reactions. Weinheim:
Wiley-VCH, 2005

[12] Ramon D J, Yus M. Angew Chem Int Ed, 2005, 44: 1602

[13] Dömling A, Wang W, Wang K. Chem Rev, 2012, 112: 3083

[14] de Graaff C, Ruijter E, Orru R V A. Chem Soc Rev, 2012, 41: 3969

[15] Duque M M S, Allais C, Isambert N, Constantieux T, Rodriguez J. Top Heterocycl Chem, 2010, 23: 227

[16] Toure B B, Hall D G. Chem Rev, 2009, 109: 4439

[17] Clarke P A, Santos S, Martin W H C. Green Chem, 2007, 9: 438

[18] Pozharskii A F, Soldatenkov A T, Katritzky A R. Heterocycles in Life and Society. 2nd ed. New York: Wiley \& Sons, 2011

[19] Sajadikhah S S, Maghsoodlou M T, Hazeri N, Habibi-Khorassani S M, Shams-Najafi S J. Monatsh Chem, 2012, 143: 939

[20] Marandi G, Hazeri N, Maghsoodlou M T, Habibi-Khorassani S M, Torbati N A, Rostami-Charati F, Skelton B W, Makhad M. J Heterocyclic Chem, 2013, 50: 568

[21] Sajadikhah S S, Maghsoodlou M T, Hazeri N. Chin Chem Lett, 2014, 25: 58

[22] Marandi G, Maghsoodlou M T, Hazeri N, Habibi-Khorassani S M, Torbati N A, Rostami-Charati F, Skelton B W, Makha M. Mol Divers, 2011, 15: 197

[23] Salahi S, Hazeri N, Maghsoodlou M T, García-Granda S, Torre-Fernández L. J Chem Res, 2014, 38: 383

[24] Lashkari M, Maghsoodlou M T, Hazeri N, Habibi-Khorassani S M, Akbarzadeh-Torbati N, García-Granda S, Torre-Fernández L. J Heterocyclic Chem, 2013, doi: 10.1002/jhet.1984

[25] Hazeri N, Lashkari M, García-Granda S, Torre-Fernández L. Aust J Chem, 2014, 67: 1656

[26] Wang P Y, Song L P, Yi H, Zhang M, Zhu S Z, Deng H M, Shao M. Tetrahedron Lett, 2010, 51: 3975

[27] Ramachary D B, Chowdari N S, Barbas C F III. Angew Chem Int Ed, 2003, 42: 4233

[28] Ramachary D B, Anebouselvy K, Chowdari N S, Barbas C F III. J Org Chem, 2004, 69: 5838

[29] Ramachary D B, Chowdari N S, Barbas C F III. Synlett, 2003: 1910

[30] Jiang B, Hao W J, Zhang J P, Tu S J, Shi F. Org Biomol Chem, 2009, 7: 2195

[31] Shults E E, Semenova E A, Johnson A A, Bondarenko S P, Bagryanskaya I Y, Gatilov Y V, Tolstikov G A, Pommier Y. Bioorg Med Chem Lett, 2007, 17: 1362

\section{Graphical Abstract}

Chin. J. Catal., 2015, 36: 1023-1028 doi: 10.1016/S1872-2067(15)60846-4

\section{An efficient green synthesis of dispirohydroquinolines via a diastereoselective one-pot eight-component reaction}

Sajjad Salahi, Malek Taher Maghsoodlou*, Nourallah Hazeri, Mojtaba Lashkari, Santiago Garcia-Granda, Laura Torre-Fernandez University of Sistan and Baluchestan, Iran; University of Oviedo-CINN, Spain
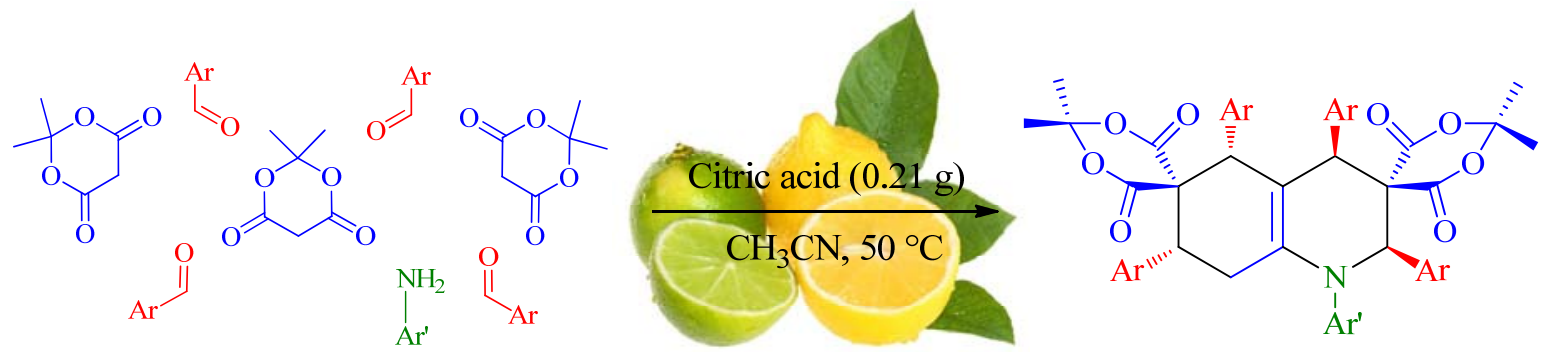

The one-pot eight-component reaction between Meldrum's acid, an aromatic aldehyde and an arylamine was achieved in the presence of citric acid as the catalyst. The corresponding dispirohydroquinoline was obtained in good yield with excellent diastereoselectivity. This method is a combination of the Knoevenagel and Michael reactions. 
[32] Shi J, Liu Y B, Wang M, Lin L L, Liu X H, Feng X M. Tetrahedron, 2011, 67: 1781

[33] Pizzirani D, Roberti M, Recanatini M. Tetrahedron Lett, 2007, 48: 7120

[34] Hao W J, Jiang B, Tu S J, Wu S S, Han Z G, Cao X D, Zhang X H, Yan S, Shi F.J Comb Chem, 2009, 11: 310

[35] Pizzirani D, Roberti M, Grimaudo S, Di Cristina A, Pipitone R M, Tolomeo M, Recanatini M. J Med Chem, 2009, 52: 6936
[36] Tu S J, Zhu X T, Zhang J P, Xu J N, Zhang Y, Wang Q, Jia R H, Jiang B, Zhang J Y, Yao C S. Bioorg Med Chem Lett, 2006, 16: 2925

[37] Wang X S, Zhang M M, Jiang H, Yao C S, Tu S J. Tetrahedron, 2007, 63: 4439

[38] Sun J, Xia E Y, Wu Q, Yan C G. ACS Comb Sci, 2011, 13: 421

[39] Ramachary D B, Barbas C F III. Chem Eur J, 2004, 10: 5323

[40] Dumas A M, Seed A, Zorzitto A K, Fillion E. Tetrahedron Lett, 2007, 48: 7072 